\title{
Design and Implementation of PM2.5 Monitoring Application Based on Android and Remote Sensing Data
}

\author{
Luo Zhang \\ State Key Laboratory of Remote Sensing Science, \\ Institute of Remote Sensing and Digital Earth, Chinese \\ Academy of Sciences \\ Computer and Information Engineering College, Henan \\ University, Kaifeng, China \\ E-mail: 190793605@qq.com \\ Feng-Bin Zheng \\ Computer and Information Engineering College, Henan \\ University, Kaifeng, China \\ E-mail: zhengfb@henu.edu.cn
}

\author{
Shu Xu \\ The School of Mathematics and Computer Science, \\ Xinyang Vocational and Technical College, Xinyang, \\ China \\ E-mail: 12550151@qq.com \\ Qiang Ge \\ Computer and Information Engineering College, Henan \\ University, Kaifeng, China \\ E-mail: gq@henu.edu.cn
}

\begin{abstract}
The traditional ground-based monitoring network have been too coarse to meet people's right to know the air pollution over current location and sounding areas, based on multi-satellite data and Android platform, an air-pollution monitoring system is designed to provide high-resolution PM2.5 concentrations information. The remote sensing results are generated through My Server and dynamically displayed by smartphone. When the pollutant concentrations exceed the standard value, the real-time alert function is activated by android message pushing mechanism. Through the experiment in Beijing areas and the comparison between satellite-retrieval and ground-based monitoring, our app has been validated with high feasibility and availability.
\end{abstract}

Keywords-air pollution monitoring; satellite remote sensing; android; pm2.5; message pushing

\section{INTRODUCTION}

PM2.5, is also known as fine particulate matter, whose aerodynamic diameter is less than or equal $2.5 \mu \mathrm{m}$ in the ambient air, can be suspended in the air for a long time [1]. Since 2013, China's environmental protection department has initially established a network consisted of ground monitoring stations. However, due to the expensive monitoring equipment and distributed site, it lead to that conventional ground-based observations can only obtain the concentrations of PM2.5 near the monitoring sites. With respect to the ground monitoring, satellite remote sensing data have the advantages of covering larger area, quasi-realtime access, dynamic updates, and low cost [2]. Currently, many of Earth observation satellites are equipped with air pollution monitoring capabilities, such as the United States NASA / EOS emission Terra, Aqua and domestic series of GF-satellites. With the continuous development of quantitative remote sensing of atmospheric algorithm, related atmospheric remote sensing products and business systems are also being gradually applied to other sectors of environmental protection, such as component-based regional haze monitoring system, which is intended for acquiring the distribution and intensity of haze pollution. J2EE-based fire point remote monitoring system can make use of satellite data to statistics and analysis of different provinces with straw burning and contamination [4].

Compared with the profession applications, the general publics have more urgent to understand the PM2.5 concentrations around them, but the traditional ground monitoring and desktop applications often cannot meet the above requirements. Currently, based on the Android platform applications become more and more popular because of its convenient, fast and so on, and many scholars have tried to develop some daily applications. For example, Gu Cong and his team members designed an indoor LBS system based on Android platform, which made it reality that users in the indoor environment can also enjoy the convenience of ubiquitous computing [5]; Guo ZhiTao and his team members develop an intelligent medical system based on Android platform, to solve the problem that using the traditional way cannot have real-time in data transmission. However, there is no domestic satellite remote sensing monitoring system developed based on Android platform. Due to some limits of the single satellite data source, like fixed transit time, return period, spatial resolution and other factors, it results in inefficient satellite data acquisition, insufficient data and other shortages, therefore, this paper tries to combine Android platform with multi-source satellite data, designs and implements a monitoring system for atmospheric pollution. 


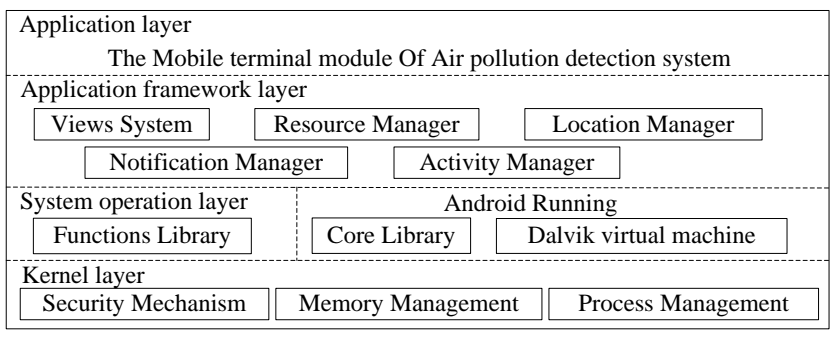

Figure 1. Framework of mobile terminal.

\section{SYSTEM ARCHITECTURE DESIGN}

The overall structure of the system includes a server and a mobile terminal, and the two separate parts interact through the network.

1) Service-terminal: Including two servers, My Server and Android Push Notification Server (Android push messaging server, referred APNS). My Server uses the open source Tomcat server to process client requests and satellite raw data, and sends the saved PM2.5 remote sensing products to database; APNS uses androidpn-servertomcat version, which is mainly responsible for pushing prompt message to the mobile terminal.

2) Mobile-terminal: Using the Android platform, by WIFI, 3G, and other ways communicate with the server through an intermediate Internet channel, receive and display the PM2.5 concentration information returned from the server. Mobile client architecture consists of four levels, the application layer, application framework layer, runtime system layer and the Linux kernel layer, as shown in Fig. 1.

a) The application layer is used to store a developed program, such as calendar, contacts, and browser and so on; it's the layer in which the PM2.5 monitoring system's mobile terminal modules built in this paper runs.

b) Application framework layer is an API framework provided by Android platform for developing applications, this system needs to call the view of the system, the location manager, event manager and other components in this layer.

c) Component libraries and virtual machine constitute the system runtime layer, the former is an important part to connect the application framework layer with the Linux kernel layer, and the latter provides a runtime environment for each Android application.

d) Linux kernel layer as an abstraction layer between hardware and software, this system's underlying layer is dependent on the core services provided by the kernel, such as system security, memory management, display drivers, network protocols, etc.

\section{SYSTEM FUNCTIONAL DESIGN}

\section{A. Server Design}

1) My server design: Aerosol optical depth (AOD), the most commonly used atmospheric parameter obtained from satellite remote sensing, stands for atmospheric aerosol particles of the entire inner layer attenuation to the visible light. The researches have shown that exist a stable relationship between aerosol optical thickness of satellite observations and particulate matter concentrations near the ground at a given condition. But the AOD-PM2.5 correlation is also affected by the vertical distribution of particulate matter and the relative humidity and other factors [7].

According to different multi-source satellite temporal resolutions, NASA dark object algorithm is exploited to retrieval the whole AOD by solving cloud recognition, aerosols and surface noise removal, with the data source derived form the newly acquired satellite raw data. On this basis, by means of the vertical distribution of aerosols and relative humidity, wind speed and other meteorological parameters provided by ground or atmospheric chemistry models and other auxiliary data, the vertical correction and absorbent correction of AOD are conducted, finally PM2.5 concentrations near the ground product of public concerned are produced[7].

To reduce network transmission time and transmission error rates, the satellite data processing system (hereinafter referred to as the processing system) is directly deployed on My Server. Server adds products to the database by sending a command. Each database record includes longitude Lon, Lat Lat, date, a corresponding place name, PM2.5 concentration value and satellite sources and other fields. Each of the latitude and longitude is unique from other records as a primary key. When the phone initiates a request, the server selects the satellite data recorded corresponded with the query criteria in the database according to Lon, Lat, the request time. If there is no satellite transit when it is requesting, the most closet record will be found. Secondly, the server judges that whether the PM2.5 concentration in the record exceeds the precedent setting standards. Finally, send the different judgment result to the message push server. The process mentioned above is shown in Fig. 2.

2) APNS design: There are many mature push ways to implement server-side push message and cell phone receives message functions. Considering the network's bandwidth and cost, this paper uses Androidpn to achieve the push message function. Firstly, client establish a persistent connection with the server through the XMPPConnection class, and then doing the registration and login authentication, finally receive the specific notifications sent by the server which based on identification information. 


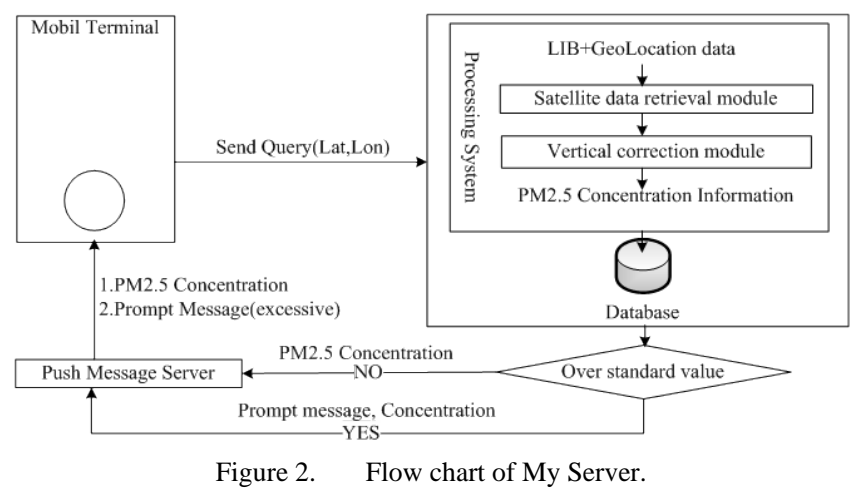

In this system, when a user issues a PM2.5 concentration query, the system's mobile terminal firstly registered through push message, and request to obtain a device token from APNS, which unique with other applications, the mobile terminal sends the device token to My Server, My Server sends the concentration values and prompt message to the APNS, APNS searches the corresponding device token which has been registered the push services in their own device list, and displays the concentration values as a $\mathrm{XML}$ form on the application whose device has right, the alert message sent to the appropriate device through the notification bar form push message way.

\section{MOBILE TERMINAL DESIGN}

Mobile terminal mainly implement to display location information returned from My Server, the PM2.5 concentration of the corresponding location and receiving messages sent by APNS and other functions, this system achieved positioning function by using Baidu map Android positioning system SDK, when the mobile originated positioning request, the positioning SDK will generate a basis according to the currently application positioning factors (GPS, base station, WIFI signal) actually situation. Specific processes are shown in Fig. 3.

Using the GeoPoint class's point object to get the user's current latitude and longitude coordinates, and mark the point on the map. In addition, design a listener interface of latitude and longitude coordinates, to ensure that users can view real-time location information of the current PM2.5 concentration, and display the monitored location information and PM2.5 concentration information on the map. This system is based on multi-source satellite data to meet the diversified needs of different users, through the high spatial resolution of satellite products, the user can zoom the map to a certain extent, view PM2.5 concentrations within a certain region. When it exceeds a different pollution levels predetermined value, the system will promptly alarm through vibration or ringing mode, and push messages, mobile terminal is responsible for receiving the message and displaying the message on the notification bar.

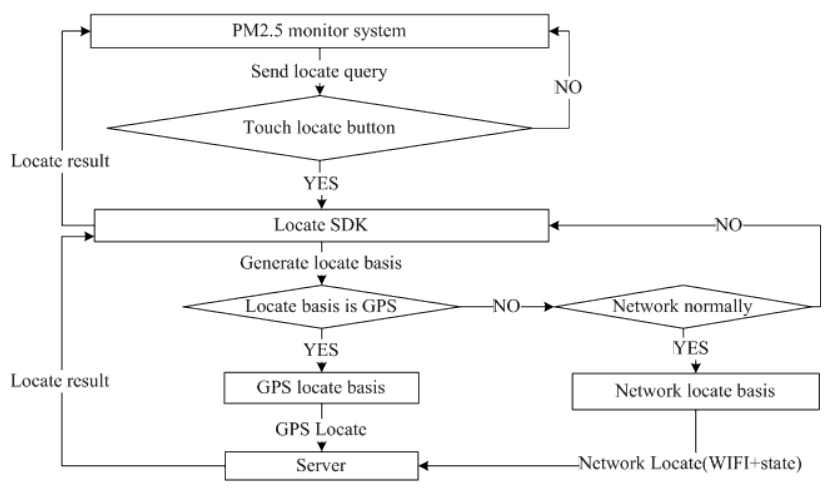

Figure 3. Flow chart of location.

TABLE I. INFORMATION OF MULTI-SATELLITES’ PARAMETERS

\begin{tabular}{|c|c|c|c|}
\hline satellite & sensor & $\begin{array}{c}\text { Product spatial } \\
\text { resolution [m] }\end{array}$ & $\begin{array}{c}\text { Observation } \\
\text { period [hour] }\end{array}$ \\
\hline Terra/Aqua & MODIS & 1000 & 12 \\
\hline NPP & VIIRS & 750 & 12 \\
\hline HJ & CCD & 30 & 48 \\
\hline GF-1 & WFV & 16 & 96 \\
\hline
\end{tabular}

\section{SYSTEM TEST}

1) Satellite data: In this paper, Terra/Aqua, Suomi NPP, HJ and GF-1 multiple satellite data as the system data source, collectively referred to named multi-source satellite data, specific parameters shown in Table 1. Terra and GF-1 transit times are the local daily time at 10:30 am, mounted on Aqua and Suomi NPP visible / infrared radiation imager VIIRS transit times are 13:30 pm, HJ-CCD transit time is 6:00 am.

In order to verify the validity of the data sources, author selects the product data which acquired from multi-source satellite in March 16, 2015, to get the day's PM2.5 satellite remote sensing maps through the processing system conversion, and compare with the traditional ground site monitoring results, as shown in Fig. 4.

The Fig. 4(a) monitored by the Beijing Environmental Protection Monitoring Center in March 16, 2015, and Fig. 4(b) obtained from the multi-source satellite monitoring inversion. As shown in Fig. 4, the number of ground monitoring stations is limited and relatively dispersed, but the amount of PM2.5 distribute information monitored by satellite is abundant. For example, only one monitoring site in Mentougou district, the distribution of PM2.5 in the block $\mathrm{A}$ and most regions throughout the central region is more complex, solely relied on the southeast site to determine the pollution around Mentougou will cause great deviation. Regional central block B in Huairou district also has the problems which mentioned above. Block $\mathrm{C}$ on behalf of the 
central region of Shunyi district, the site monitored PM2.5 concentration within the $150-250 \mu \mathrm{g} / \mathrm{m}^{3}$ range (Fig. 4(a)), but in Fig. 4(b) the concentration in this region monitored by satellite is more than $250 \mu \mathrm{g} / \mathrm{m}^{3}$.

2) Application example: The author accordingly to deploys and configures the server and the client, and tested in Beijing to verify the feasibility of the system. Specific configuration parameters: server running under Windows platform, database and My Server server on the same PC, My Server and APNS deployed on Tomcat; Client with support for Android 4.4 version of xiaomi phone.

Open APP, view the PM2.5 pollution circumstances in certain location and surrounding area. As shown in Fig. 5(a), the current position of PM2.5 concentrations is up to
$267 \mu \mathrm{g} / \mathrm{m}^{3}$, it is a serious pollution according to Chinese air quality standards, the system will alerts user the serious pollution of the current location, and should pay attention to make protective measures (Fig. 5(b)). As shown in Fig. 5(c), view the pollution of the current location and the surrounding $1.1 \mathrm{~km}$ away of each location. It is not difficult to find that the pollution values of the Institute of Geographic where located on the side of the road is higher than the other four places, the Olympic Village where close to the Forest Park is relatively low, but all the five places the PM2.5 concentrations are $168 \mu \mathrm{g} / \mathrm{m}^{3}$ monitored by the traditional ground stations, thus proved that satellite remote sensing can provide more precise information on air pollution.
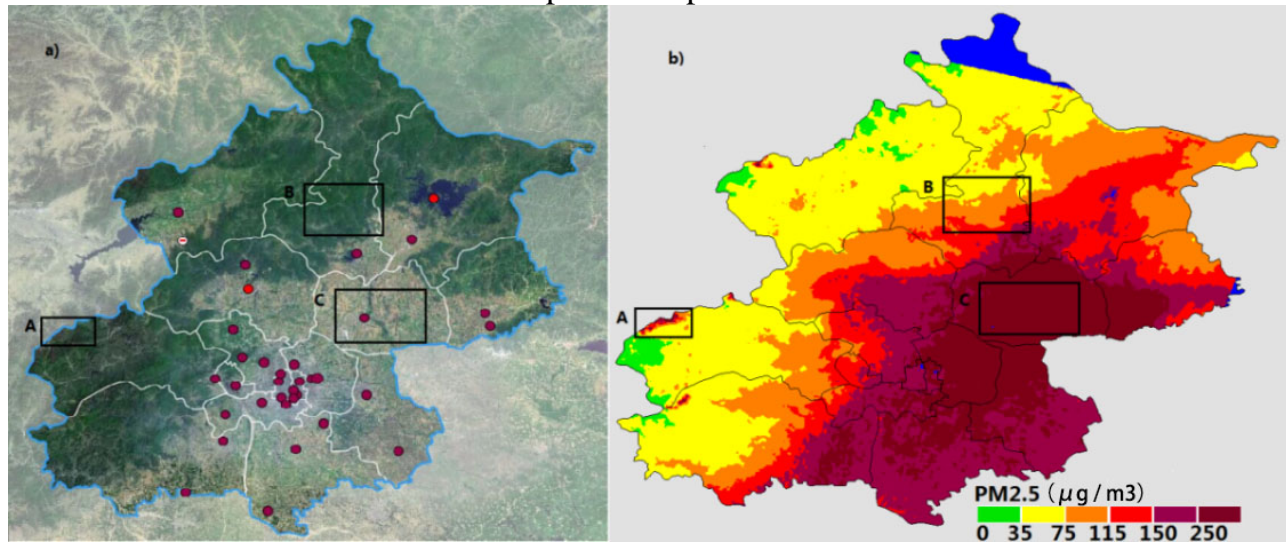

Figure 4. Comparison between monitoring sites and remote sensing monitoring in Mar.16, 2015: (a) Beijing Environmental Protection Monitoring Center monitoring in March 16, 2015; (b) Multi-source satellite monitoring renderings.

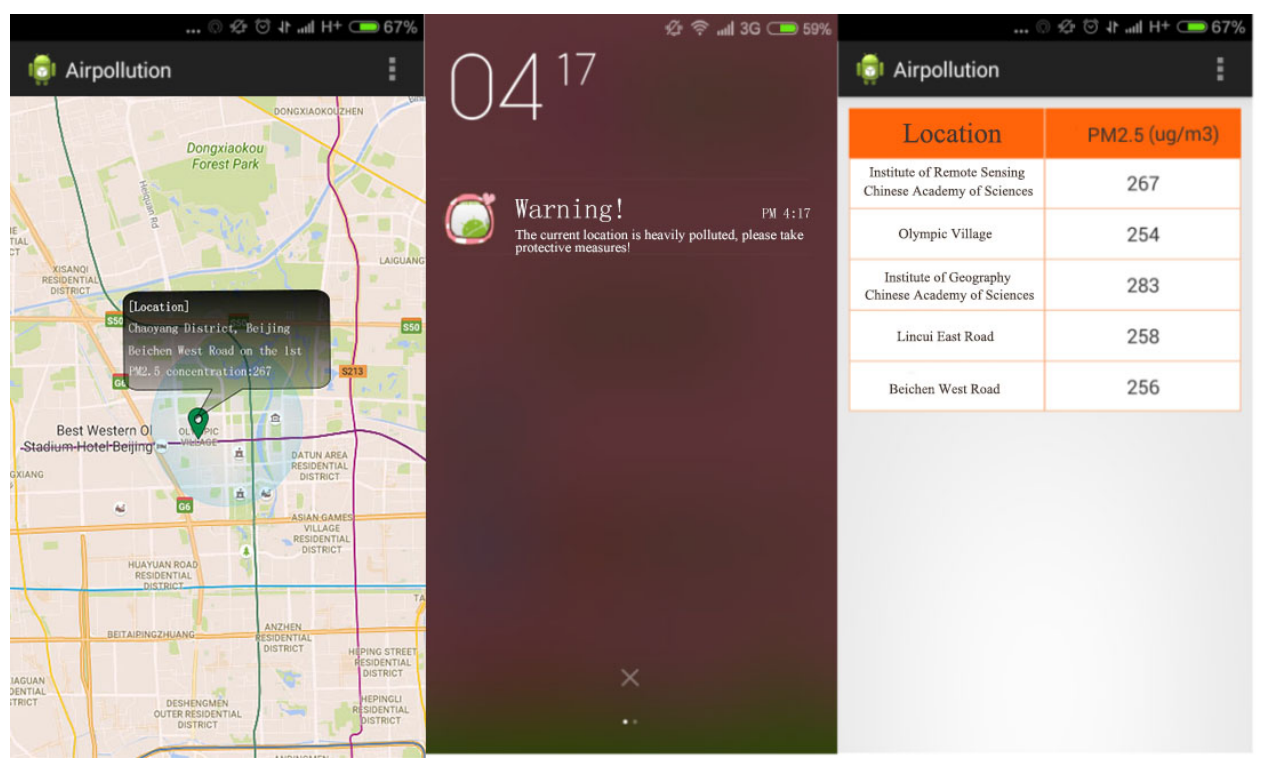

Figure 5. Tested graph in Beijing, Mar. 16, 2015: (a) Locate and request PM2.5 concentration; (b) Message alerts; (c) A PM2.5 concentration list of around location

\section{ACKNOWLEDGEMENT}

This paper describes the design concept, overall structure, core functions and results display of this PM2.5 monitoring system, using My Server and APNS two servers, respectively to achieve the production of satellite remote sensing, processing client requests and messages push functions. Compared to the current air monitoring and 
forecasting mode, the system enables to let user initiative to learn the location and the surrounding area air quality situation. However, due to limitations of satellite data reception time delay, it is still cannot fully realized the request of real-time view of air quality, the future will consider to combine the Institute of Remote Sensing's Miyun, Sanya, Kashi three satellite receiving systems, and assimilation ground monitoring, atmospheric chemistry model data to further enrich the data sources, compensate for the inversion problem when there are clouds, thus speeding up the system's operational and application.

\section{REFERENCES}

[1] YANG Hong-bin, ZOU Xu-dong, WANG Hong-yu, et al. Study progress on PM2.5 in atmospheric environment[J]. Journal of Meteorology and Environment, 2012, 28 (3):77-82.

[2] TAN Qu-lin,SHAO Yun.Application of Remote Sensing Technology to Environmental Pollution Monitoring[J]. Remote Sensing Technology and Application, 2000, 4(15):246-251.

[3] LI Shen-shen, CHEN Liang-fu, WANG Zhong-ting, et al. Regional Haze Monitoring System Based on Component and Its Retrieval Algorithm[J]. Computer Engineering, 2009, 35(16):23-24, 28.
[4] HE Bao-hua, CHEN Liang-fu, Li Shen-shen, et al. Design of Straw Fire Remote Sensing Monitoring System Bases on J2EE[J]. Microcomputer Information, 2010, 26 (4-3):38-39.

[5] GU Cong, CHEN Yi-qing, LIU Jun-fa, et al. Design of indoor LBS system based on anddroid platform and its Implementation[J]. Computer Engineering and Design, 2012, 33(1):396-401.

[6] GUO Zhi-tao, GUO Zhong, WANG Zhen. Design and Realization of Intelligent Medical System Based on Android Platform[J]. Journal of Zhengzhou University(Engineering Science), 2014, 35(6):60-63.

[7] WANG Zi-feng, CHEN Liang-fu, TAO Jin-hua, et al. Satellite-based estimation of regional particulate matter(PM) in vertical-and RH correcting method[J]. Remote Sensing of Environment, 2010, 114(1):50-63.

[8] KAUFMAN Y J, WALD A E, REMER L A, et al. The MODIS 2.1um channel-correlation with visible reflectance for use in remote sensing of aerosol[J]. IEEE Transactions on Geoscience and Remote Sensing, 1997, 35(5):1286-1298.

[9] Li S.S., Chen L.F.,Tao J.H., et al. Retrieval of aerosol optical depth over bright targets in the urban areas of North China during winter. China Science, 2012, 42(8):1253-1263.

[10] FU Sha. The research and implementation of enterprise instant messaging system based on XMPP[D]. Southwest Jiaotong University School of Information, 2009. 